COMMENTARY

\title{
Targeting branch sites of new exons?
}

\author{
Igor Vorechovsky
}

\section{Correspondence to}

Dr Igor Vorechovsky, HDH,

University of Southampton,

MP808, Southampton S016

6YD, UK; igvo@soton.ac.uk

Accepted 18 May 2012

Published Online First

22 June 2012
Martinez et $a l^{1}$ reported an interesting case of Dubowitz-like syndrome associated with an acceptor splice site mutation, leading to the use of a cryptic splice donor site in an Alu sequence. Their explanation for the resulting aberrant transcripts was limited to a referral to Alu polyA tails and Aluderived microRNAs. ${ }^{1}$ Upon mutation of acceptor sites, the spliceosome machinery tries to find suitable alternative $3^{\prime}$ splice sites nearby. If none is available, which is the most likely scenario the downstream exon(s) is skipped or the mutated intron is retained in mature transcripts. If there is a suitable replacement in the pre-mRNA, the mutation may activate the upstream or downstream cryptic acceptor site(s) and still employ the authentic donor counterpart. ${ }^{2}$ This was not the case in the reported family. The new donor splice site was weak, with a maximum entropy score of 6.79 , which is below the average for natural $5^{\prime}$ splice sites. ${ }^{2}$ Clearly, the mutation activated an ideal cryptic $3^{\prime}$ splice site in a downstream Alu element. ${ }^{1}$ However, this transposon is in the antisense orientation in NSUN2 intron 6 (ref. 1) and does not therefore contain a polyA as the authors presented, but contains a polyuracil tail. This motif serves as a powerful $3^{\prime}$ splice site activation motif in vertebrates, termed the polypyrimidine tract, with uracils providing generally superior signals over cytosines. ${ }^{3}$ The polyuracil tracts in Alus critically influenced their exonisation in primates, contributing to new exon birth and evolutionary success of alternative splicing. ${ }^{4}$ Thus, the defect in this family recapitulated an ancient RNA processing event that occurred many times in primate evolution, improved the fine-tuning of gene expression and expanded proteomic diversity.

However, even the optimal 3' splice site may not be sufficient for high-inclusion exonisation and overt phenotype unless additional splicing signals are present in primary transcripts, including a suitable branch site. I inspected the reference gene sequence just upstream of the polyuracil tract and found five adjacent adenines that would serve as a source of this preferred nucleobase for branch point recognition, and putative base-pairing interactions that contribute to the required pre-mRNA structure of the chosen acceptor. Using a branch point prediction tool, ${ }^{6}$ this adenine stretch exactly co-localised with the predicted branch point sequence of the new exon. Although not applicable in this particular family, correction of cryptic exon activation by antisense oligonucleotides may therefore be further improved by co-targeting branch sites of new exons along with their splice sites and splicing enhancers.

Contributors I certify that neither this manuscript nor one with substantially similar content under my authorship has been published or is being considered for publication elsewhere.

\section{Competing interests None.}

Provenance and peer review Not commissioned; internally peer reviewed.

\section{REFERENCES}

1. Martinez FJ, Lee JH, Lee JE, et al. Whole exome sequencing identifies a splicing mutation in NSUN2 as a cause of a Dubowitzlike syndrome. J Med Genet. 2012;49:380-85.

2. Buratti $\mathbf{E}$, Chivers MC, Hwang G, et al. DBASS5: databases of aberrant $3^{\prime}$ and $5^{\prime}$ splice sites in human disease genes. Nucleic Acids Res 2011;39:D86-91

3. Bouck J, Fu XD, Skalka AM, et al. Genetic selection for balanced retroviral splicing: novel regulation involving the second step can be mediated by transitions in the polypyrimidine tract. Mol Cell Biol 1995;15:2663-71.

4. Sorek R, Ast G, Graur D. Alu-containing exons are alternatively spliced. Genome Res 2002:12:1060-7.

5. Lev-Maor G, Sorek R, Shomron N, et al. The birth of an alternatively spliced exon: $3^{\prime}$ splice-site selection in Alu exons. Science 2003;300:1288-91.

6. Kol G, Lev-Maor G, Ast G. Human-mouse comparative analysis reveals that branch-site plasticity contributes to splicing regulation. Hum Mol Genet 2005;14:1559-68.
This paper is freely available online under the BMJ Journals unlocked scheme, see http:// jmg.bmj.com/site/about/ unlocked.xhtml 